\title{
Usefulness of Contrast-Enhanced 3D-FLAIR MR Imaging for Differentiating Rathke Cleft Cyst from Cystic Craniopharyngioma
}

\author{
(D) M. Azuma, (D) Z.A. Khant, (D) M. Kitajima, (D). Uetani, (D). Watanabe, (D) K. Yokogami, (D). Takeshima, and (D). Hirai
}

\begin{abstract}
BACKGROUND AND PURPOSE: Because it can be difficult to discriminate between a Rathke cleft cyst and cystic craniopharyngioma by conventional MR imaging alone, we investigated whether contrast-enhanced 3D T2-FLAIR MR imaging at 3T helps to distinguish a Rathke cleft cyst from a cystic craniopharyngioma.
\end{abstract}

MATERIALS AND METHODS: We evaluated pre- and postcontrast T1-weighted and 3D T2-FLAIR images of 17 patients with pathologically confirmed Rathke cleft cyst $(n=10)$ or cystic craniopharyngioma $(n=7)$. All underwent 3T MR imaging studies before surgery. Two neuroradiologists independently recorded the enhancement grade of the lesion wall as grade 2 (most of the wall enhanced), grade 1 (some of the wall enhanced), and grade 0 (none of the wall enhanced). One neuroradiologist performed a blinded reading study of conventional MR images with/without 3D T2-FLAIR images. Interobserver agreement was determined by calculating the $\kappa$ coefficient. Statistical analyses, including receiver operating characteristic curve analysis were performed.

RESULTS: Interobserver agreement for postcontrast TIWI and 3D T2-FLAIR images was excellent $(\kappa=0.824$ and $\kappa=0.867$, respectively). Although the difference in the mean enhancement grade of Rathke cleft cysts and cystic craniopharyngiomas was not significant on postcontrast TIWIs, it was significant on postcontrast 3D T2-FLAIR images $(P=.0011)$. The area under the receiver operating characteristic curve of the conventional MR alone and conventional MR with 3D T2-FLAIR readings was 0.879 and 1.0, respectively, though there was no significant difference in the area under the curve between the 2 readings.

CONCLUSIONS: Contrast-enhanced 3D T2-FLAIR imaging at 3T helps to distinguish a Rathke cleft cyst from cystic craniopharyngioma.

ABBREVIATIONS: $\mathrm{CCP}=$ cystic craniopharyngioma; $\mathrm{CE}=$ contrast-enhanced; $\mathrm{RCC}=$ Rathke cleft cyst

A Rathke cleft cyst (RCC) with mild symptoms can be managed conservatively because it may shrink or disappear spontaneously. ${ }^{1}$ Severe symptoms are usually managed by surgical drainage with partial excision of the cyst wall. ${ }^{2}$ Cystic craniopharyngioma (CCP), however, usually requires more extensive surgery and/or radiation therapy. ${ }^{3}$ Therefore, the differentiation between an RCC and a CCP is important.

The discrimination between RCC and CCP on CT and conventional MR imaging can be difficult. ${ }^{4}$ According to Hofmann et $\mathrm{al}^{5}{ }^{5}$ the size of the lesion $(>2 \mathrm{~cm})$, its suprasellar location, and

Received August 16, 2019; accepted after revision October 29.

From the Departments of Radiology (M.A., Z.A.K., T.H.) and Neurosurgery (T.W., K.Y., H.T.), Faculty of Medicine, University of Miyazaki, Miyazaki, Japan; and Department of Diagnostic Radiology (M.K., H.U.), Graduate School of Medical Science, Kumamoto University, Kumamoto, Japan.

This study was supported by a Grant-in-Aid for Clinical Research from Miyazaki University Hospital.

Please address correspondence to Minako Azuma, Department of Radiology, Faculty of Medicine, University of Miyazaki, 5200 Kihara, Kiyotake, Miyazaki

889-1692, Japan; e-mail: minako_azuma@med.miyazaki-u.ac.jp

http://dx.doi.org/10.3174/ajnr.A6359

the presence of calcification were diagnostic for CCP. Choi et $\mathrm{al}^{6}$ reported that superior tumor lobulation, a large tumor volume, and compression of the third ventricle on MR imaging supported a diagnosis of CCP. However, Choudhry et $\mathrm{al}^{7}$ encountered an RCC with CCP features, that is, a large lesion size $(6.5 \mathrm{~cm})$, suprasellar location, calcification, superior tumor lobulation, and compression of the third ventricle. Hua et $\mathrm{al}^{4}$ suggested that cyst wall enhancement may help to differentiate neoplastic from non-neoplastic cystic lesions. However, many RCCs are surrounded by the enhancing normal pituitary gland and this mimics wall enhancement, ${ }^{8}$ which renders the differentiation between RCC and CCP difficult.

The contrast-enhanced (CE) 3D T2-FLAIR MR imaging sequence is more highly sensitive to low concentrations of gadolinium than $\mathrm{T}_{\mathrm{WWI}}{ }^{9,10}$ and the signal intensity of vessels at a flow velocity that exceeds $1.0 \mathrm{~cm} / \mathrm{s}$ is suppressed. ${ }^{11}$ On CE 3D T2FLAIR images, the anterior lobe of the pituitary gland is often unenhanced. ${ }^{12}$ Therefore, we investigated whether differences in cyst wall enhancement on CE 3D T2-FLAIR MR imaging help to differentiate between an RCC and a CCP. 


\section{Contrast-enhanced T1WI}

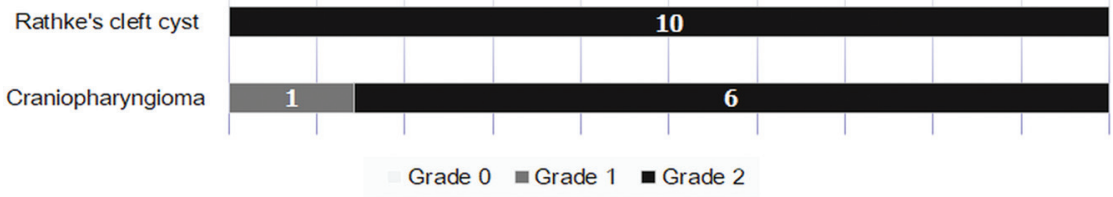

\section{Contrast-enhanced 3D T2-FLAIR}

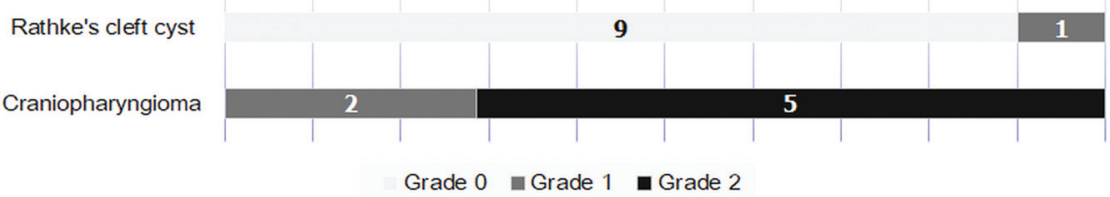

FIG 1. Distribution of the wall-enhancement grade of RCC and CCP on CE TIWI and 3D T2-FLAIR images.

\section{MR Study Protocol}

All studies were performed on a $3 \mathrm{~T}$ MR imaging scanner (Magnetom Trio; Siemens, Erlangen, Germany); a 12channel head coil was used. The scanning parameters for 3D T2-FLAIR were TR, $6000 \mathrm{~ms}$; TEeff, $420 \mathrm{~ms}$; inversion time, $2000 \mathrm{~ms}$; echo-train length, 142; imaging time, $4 \mathrm{~min}$ $26 \mathrm{~s}$; field of view, $230 \times 230$ $\mathrm{mm}$; matrix, $256 \times 256$; and 0.9 -mm-thick sections. For T1WI, the parameters were TR, $560 \mathrm{~ms}$; TE, $11 \mathrm{~ms}$; section thickness, $3 \mathrm{~mm}$; matrix, $256 \times 218$; field of view, $130 \mathrm{~mm}$ on coronal and sag-

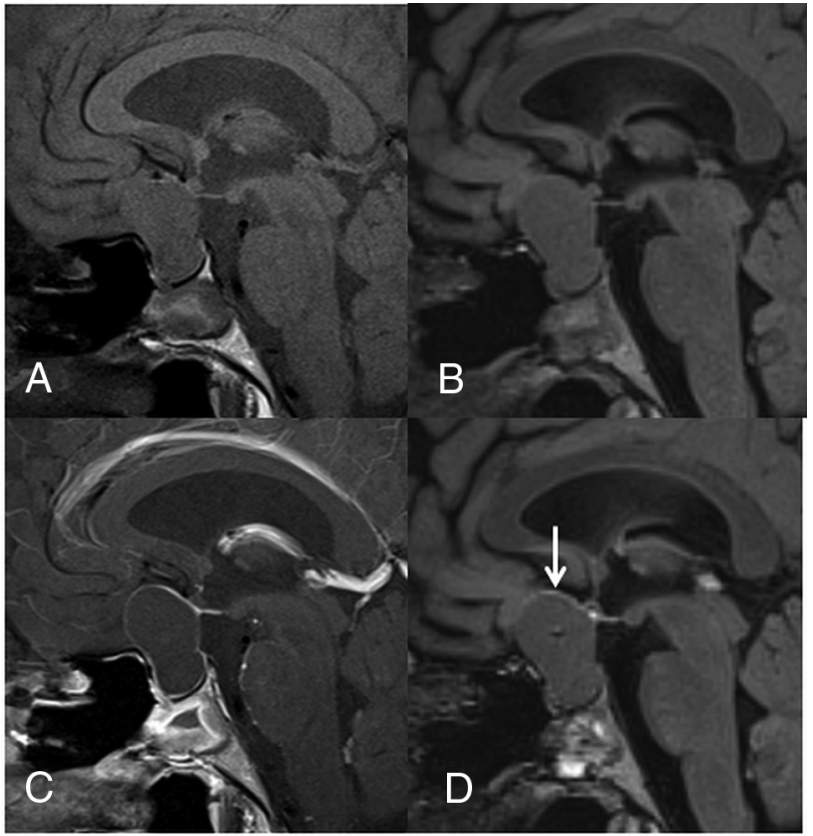

FIG 2. A 23-year-old woman with RCC. Compared with the precontrast TIWI $(A)$, the postcontrast TIWI $(C)$ demonstrates enhancement in most of the cyst wall (grade 2). Compared with the precontrast 3D T2-FLAIR image $(B)$, the postcontrast 3D T2-FLAIR image $(D)$ shows grade 1 enhancement of some of the cyst wall (arrow). In the blinded reading study, the confidence scale of this lesion was probably an RCC (scale 2) at both the first and second interpretation sessions.

\section{MATERIALS AND METHODS \\ Study Population}

Our retrospective study was approved by the institutional review board of the University of Miyazaki Hospital; informed consent was waived. Seventeen consecutive patients ( 9 men, 8 women; age range, 23-81 years; mean age, 51.6 years) were included: 10 had pathologically confirmed RCC, the other 7 had CCP. Before surgery, all had undergone conventional MR pre- and postcontrast T1WI and 3D T2-FLAIR imaging studies on a $3 \mathrm{~T}$ unit. ittal planes. The contrast agent was gadopentetate dimeglumine (Magnevist, Bayer HealthCare Pharmaceuticals, Wayne, New Jersey), injected at $0.1 \mathrm{mmol} / \mathrm{kg}$ body weight. To reduce the imaging time, we obtained sagittal 3D planes that covered the entire brain with each pulse sequence. CE MR studies were started approximately 60-120 s after contrast material injection; a T1-weighted sequence was the first sequence.

\section{Image Evaluation}

Two neuroradiologists with 6 and 8 years of reading experience who were blinded to the patient identity and the final diagnosis independently graded the degree of CE of the cyst wall on T1WI and 3D T2-FLAIR images on a PACS workstation by using a 3point scoring system in which grade 2 represented most of the wall enhanced, grade 1 represented some of the wall enhanced, and grade 0 represented no wall enhancement. Final judgments were obtained by consensus of the 2 readers.

One experienced neuroradiologist with 28 years of reading experience performed a blinded reading study. Each case was subjected to 2 reading sessions on a PACS workstation. At the first session, the reader was provided with only the conventional MR images to diagnose the 2 types of pituitary lesions. Sagittal views of pre- and postcontrast T1WI, and T2WI were evaluated. The reader assessed the lesions by using a 5-point confidence scale: 1 , definitely an RCC; 2 , probably an RCC; 3 , equivocal; 4 , probably a CCP; and 5, definitely a CCP. After the first session, the reader performed a second interpretation in which sagittal views of pre- and postcontrast 3D T2-FLAIR images were added. The reader reassessed the lesion by using the same 5-point confidence scale. After the blinded study, the reader retrospectively identified the reason(s) for the incorrect image interpretation.

\section{Statistical Analysis}

All statistical analyses were performed with MedCalc version 19.0.7 (MedCalc Software, Mariakerke, Belgium). The statistical significance of scoring differences was analyzed by using the Mann-Whitney $U$ test. Interobserver agreement was determined by calculating the $\kappa$ coefficient in which $\kappa=0.81-1.0$ indicated 


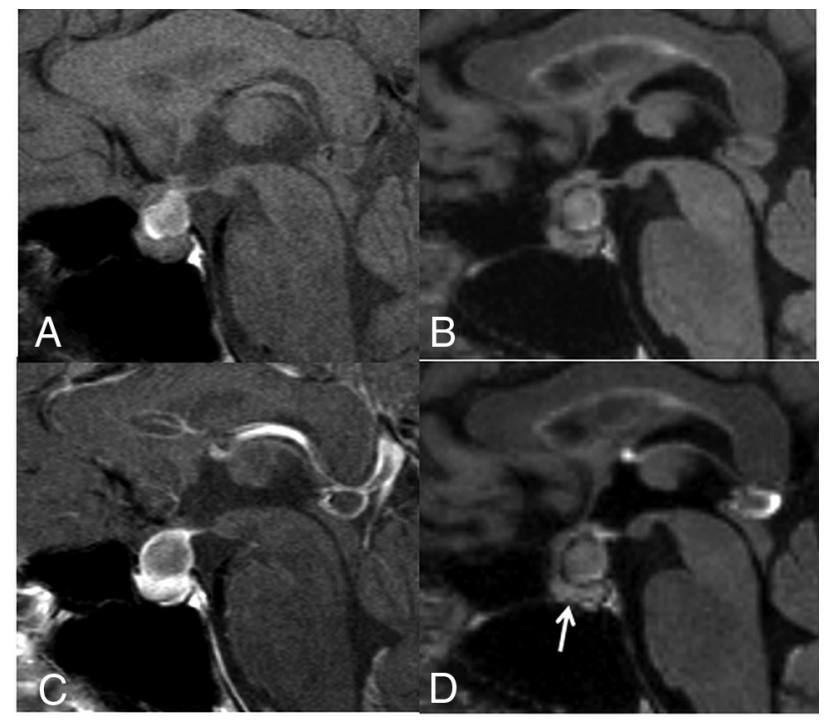

FIG 3. A 40-year-old woman with an RCC. On the postcontrast TTWI (C) compared with the precontrast TTWI $(A)$, the cyst wall is enhanced (grade 2), as is the pituitary gland. Unlike the precontrast 3D T2-FLAIR image $(B)$, the postcontrast image $(D)$ shows no contrast enhancement (grade 0 ). Different from the postcontrast TIWI $(C)$, the pituitary gland is not enhanced on the postcontrast 3D T2-FLAIR image (arrow in D). The observer judged this lesion as equivocal (scale 3) at the conventional MR interpretation, whereas the confidence level was changed to probably an RCC (scale 2) by the addition of 3D T2-FLAIR interpretation.

excellent agreement, $0.61-0.80$ indicated good agreement, $0.41-$ 0.60 indicated moderate agreement, $0.21-0.40$ indicated fair agreement, and 0-0.20 indicated slight agreement.

Receiver operating characteristic analysis was performed to evaluate the diagnostic performance of conventional MR images with and without 3D T2-FLAIR images. Lesions with a confidence score of 3, 4, or 5 were considered to be CCPs and those with a score of 1 or 2 were considered to be RCCs. Pair-wise comparison of the receiver operating characteristic curves was also performed by using the area under the receiver operating characteristic curve. Differences of $P<.05$ were considered statistically significant.

\section{RESULTS}

Interobserver agreement for postcontrast T1WI and 3D T2FLAIR images was excellent $(\kappa=0.824$ and $\kappa=0.867$, respectively). The distribution of the wall-enhancement grade of 10 RCCs and 7 CCPs on CE T1WI and CE 3D T2-FLAIR images is shown in Fig 1. On CE T1WI, all RCCs and CCPs were classified as grade 1 or 2 (Figs 2-5). The difference in the mean grade assigned to CCPs and RCCs was not significant. On CE 3D T2FLAIR images, 1 RCC was classified as grade 1 (Fig 2) and the other 9 RCCs as grade 0 (Figs 3 and 4). Of the 7 CCPs, 5 were of enhancement grade 2 (Fig 5), the others were of grade 1 . The difference in the mean grade assigned to RCCs and CCPs was significant $(P=.0011)$. As shown in Fig 6, adding the 3D T2-FLAIR images at the first reading session resulted in achieving perfect diagnostic performance in the discrimination between an RCC and a CCP. The area under the curve of the first and second

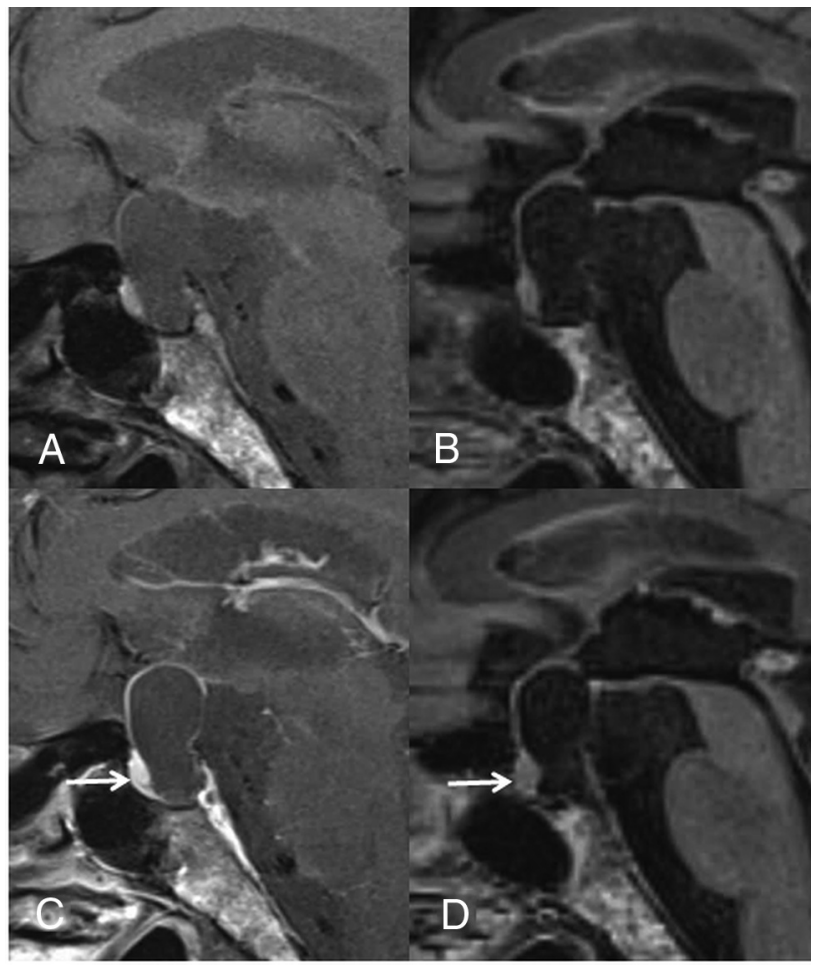

FIG 4. A 60-year-old man with RCC. On the postcontrast TIWI (C) compared with the precontrast TIWI $(A)$, the cyst wall is enhanced (grade 2) and a solid enhanced area is seen (arrow). Unlike the precontrast 3D T2-FLAIR image (B), the postcontrast image $(D)$ shows no contrast enhancement of the wall (grade 0) and the solid area (arrow). The observer judged this lesion as probably a CCP (scale 4) at the conventional MR interpretation, whereas the confidence level was changed to probably an RCC (scale 2) by the addition of 3D T2-FLAIR interpretation.

reading session was 0.879 and 1.0 , respectively. There was no significant difference in the area under the curve between the first and second reading session $(P=.16)$.

A retrospective review revealed the reasons for incorrect interpretation for 2 of $17(11.8 \%)$ conventional MR readings (first interpretation session). In one patient with an RCC, the lesion determination on conventional MR interpretation was a falsepositive result due to the presence of a solid component on $\mathrm{CE}$ T1WI (Fig 4), which was correctly interpreted as normal anterior pituitary gland by adding the 3D T2-FLAIR images (second interpretation session). In 1 patient with a CCP, the false-negative result of a conventional $\mathrm{MR}$ interpretation was attributed to a homogeneously enhanced cystic wall (Fig 5).

\section{DISCUSSION}

We found that cyst wall enhancement on CE 3D T2-FLAIR images was significantly different between an RCC and a CCP. In addition, adding CE 3D T2-FLAIR images to conventional MR images was useful for differentiating RCC from CCP. On CE T1WI, all RCCs and CCPs showed wall enhancement. On CE 3D T2-FLAIR images, however, the cyst wall enhanced in all the CCPs and in only 1 RCC. The difference in cyst wall enhancement must have contributed to our blinded interpretation results. To our knowledge, our study is the first documentation that CE 


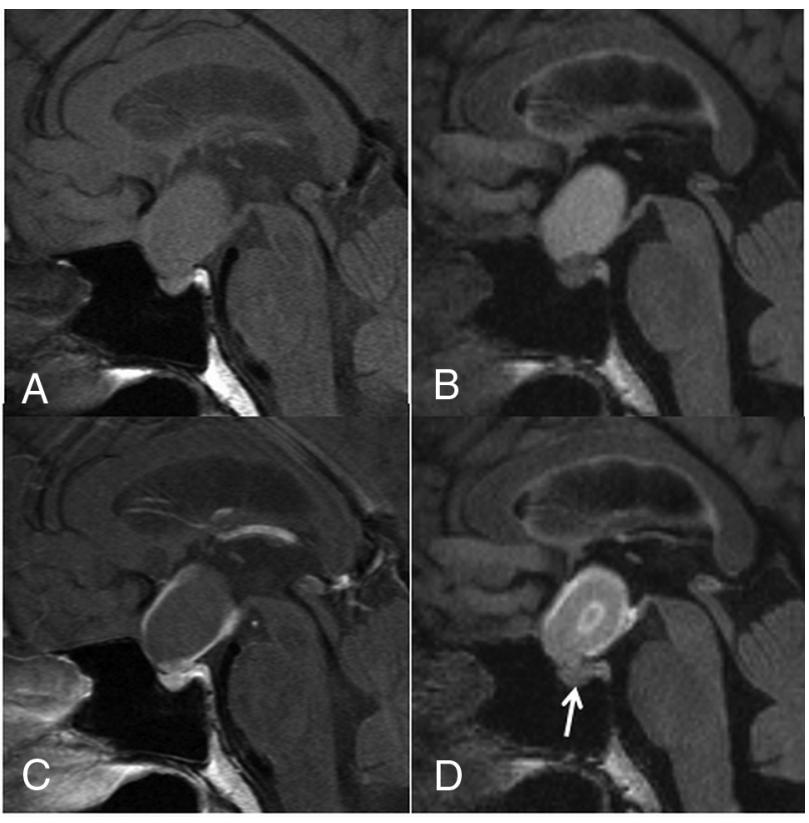

FIG 5. A 44-year-old woman with a CCP. A comparison of pre- $(A)$ and postcontrast TIWI (C) shows that the cyst wall is enhanced on the postcontrast image (grade 2). A comparison of pre- (B) and postcontrast 3D T2-FLAIR images $(D)$ reveals enhancement of the cyst wall (grade 2) and the lesion center on the postcontrast image $(D)$. Different from the postcontrast TIWI (C), the pituitary gland is not intensely enhanced on the postcontrast 3D T2-FLAIR image (arrow in $D$ ). The observer judged this lesion as probably an RCC (scale 2) at the conventional MR interpretation, whereas the confidence level was changed to probably a CCP (scale 4) by the addition of 3D T2-FLAIR interpretation.

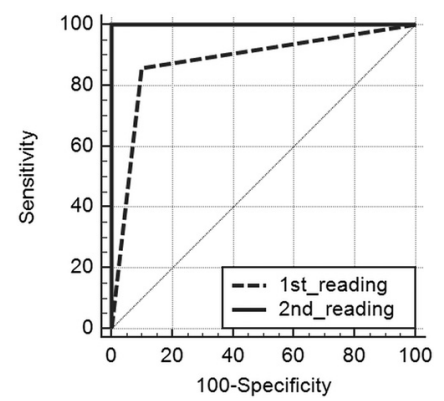

FIG 6. Comparison of the receiver operating characteristic (ROC) curves of the first (conventional MR alone) and second (conventional $M R$ and 3D T2-FLAIR) reading session. The areas under the ROC curve (AUC) of the first and second reading sessions were 0.879 and 1.0, respectively. There was no significant difference in the AUC between the first and second reading session $(P=.16)$.

3D T2-FLAIR imaging is useful for the differentiation between an RCC and a CCP.

RCCs are often surrounded by the enhancing normal pituitary gland and thus mimic wall enhancement on CE T1WI. ${ }^{8}$ On CE 3D T2-FLAIR images, the anterior lobe of the pituitary gland is often unenhanced. This may be attributable to T2 shortening due to a high gadolinium concentration or to the sequence's sensitivity to the flow in the hypophyseal portal system, which is composed of sinusoidal capillaries in the anterior pituitary gland. ${ }^{12}$ Thus, the lack of wall enhancement of the anterior pituitary gland on CE 3D T2-FLAIR images may render the differentiation of neoplastic and non-neoplastic cystic lesions possible.

$\mathrm{CE}$ in the CNS on T1-weighted sequences is the result of a combination of 2 primary processes, that is, intravascular (vascular) and interstitial (extravascular) enhancement. ${ }^{13,14}$ Enhancement on 3D-FLAIR sequences, however, is probably due to vessel permeability, which results from extravascular enhancement and suppression of the signal in the vessels. ${ }^{12}$ The wall of all CCPs was enhanced on CE T1WI and CE 3D FLAIR scans. With respect to RCCs, the wall was enhanced on all T1WI, whereas only 1 lesion wall was enhanced on 3D T2-FLAIR images. We think that the permeability of the CCP wall is high, whereas the RCC wall is less permeable. Consequently, on CE 3D T2-FLAIR sequences, the differences in the wall permeability of RCCs and CCPs resulted in helping to differentiate between the 2 types of pituitary lesions. Azuma et $\mathrm{al}^{12}$ reported enhancement of the pituitary stalk on CE 3D T2-FLAIR images. The partial enhancement of the RCC wall that we observed in 1 case on 3D T2-FLAIR images may have been due to displacement of the pituitary stalk. Another possible mechanism of the enhancement of the RCC wall may be related to inflammation or squamous metaplasia. ${ }^{15}$

The visual evaluation of sellar cystic lesions requires the acquisition of thin imaging slices. Because 3D T2-FLAIR imaging suppresses flow artifacts from $\mathrm{CSF}^{16,17}$ and yields $3 \mathrm{D}$ volume data with isotropic information, thinner section images can be acquired in any plane. This minimizes the partial volume effect between small lesions and surrounding tissue, ${ }^{18,19}$ and these advantages of CE 3D T2-FLAIR imaging were useful in our study.

Our study had some limitations. Although our study population was small, our findings were not unimportant and encourage clinical studies on larger populations. To decrease the influence of partial volume effects on our interpretation of the images, we used 0.9- and 3-mm isovoxel images in our assessment of 3D T2FLAIR images and T1WI, respectively. Also, the time-dependence of gadolinium enhancement may have affected our results. The first CE sequence that we acquired in all instances was the conventional T1-weighted sequence; because the $3 \mathrm{D}$ sequence was obtained subsequently, we may have overestimated the added value of the $3 \mathrm{D}$ sequence.

\section{CONCLUSIONS}

On CE 3D FLAIR images, RCCs and CCPs showed significantly different wall enhancement; consequently, we suggest that CE 3D FLAIR images are useful for differentiating between RCC and CCP.

Disclosures: T. Hirai-RELATED: Grant: Grant-in-Aid for Clinical Research from the University of Miyazaki Hospital.

\section{REFERENCES}

1. Saeki N, Sunami K, Sugaya Y, et al. MRI findings and clinical manifestations in Rathke's cleft cyst. Acta Neurochir (Wien) 1999;141:1055-61 CrossRef Medline

2. Kim JE, Kim JH, Kim OL, et al. Surgical treatment of symptomatic Rathke cleft cysts: clinical features and results with special attention to recurrence. J Neurosurg 2004;100:33-40 CrossRef Medline 
3. Müller HL. Risk-adapted, long-term management in childhood-onset craniopharyngioma. Pituitary 2017;20:267-81 CrossRef Medline

4. Hua F, Asato R, Miki Y, et al. Differentiation of suprasellar nonneoplastic cysts from cystic neoplasms by Gd-DTPA MRI. J Comput Assist Tomogr 1992;16:744-49 CrossRef Medline

5. Hofmann BM, Kreutzer J, Saeger W, et al. Nuclear beta-catenin accumulation as reliable marker for the differentiation between cystic craniopharyngiomas and Rathke cleft cysts: a clinico-pathologic approach. Am J Surg Pathol 2006;30:1595-603 CrossRef Medline

6. Choi SH, Kwon BJ, Na DG, et al. Pituitary adenoma, craniopharyngioma, and Rathke cleft cyst involving both intrasellar and suprasellar regions: differentiation using MRI. Clin Radiol 2007;62:45362 CrossRef Medline

7. Choudhry OJ, Choudhry A, Patel SK, et al. Giant suprasellar Rathke's cleft cyst mimicking craniopharyngioma: implications for a spectrum of cystic epithelial lesions of ectodermal origin. $J$ Neurol Surg A Cent Eur Neurosurg 2012;73:324-29 CrossRef Medline

8. Byun WM, Kim OL, Kim D. MR imaging findings of Rathke's cleft cysts: significance of intracystic nodules. AJNR Am J Neuroradiol 2000;21:485-88 CrossRef Medline

9. Deliganis AV, Fisher DJ, Lam AM, et al. Cerebrospinal fluid signal intensity increase on FLAIR MR images in patients under general anesthesia: the role of supplemental O2. Radiology 2001;218:152-56 CrossRef Medline

10. Maeda M, Tsuchida C. "Ivy sign" on fluid-attenuated inversionrecovery images in childhood moyamoya disease. AJNR Am J Neuroradiol 1999;20:1836-38 Medline

11. Fukuoka H, Hirai T, Okuda T, et al. Comparison of the added value of contrast-enhanced 3D fluid-attenuated inversion recovery and magnetization-prepared rapid acquisition of gradient echo sequences in relation to conventional postcontrast T1-weighted images for the evaluation of leptomeningeal diseases at 3T. AJNR Am J Neuroradiol 2010;31:868-73 CrossRef Medline

12. Azuma M, Hirai T, Kadota $Y$, et al. Circumventricular organs of human brain visualized on post-contrast 3D fluid-attenuated inversion recovery imaging. Neuroradiology 2018;60:583-90 CrossRef Medline

13. Provenzale JM, Mukundan S, Dewhirst M. The role of blood-brain barrier permeability in brain tumor imaging and therapeutics. $A J R$ Am J Roentgenol 2005;185:763-67 CrossRef Medline

14. Smirniotopoulos JG, Murphy FM, Rushing EJ, et al. Patterns of contrast enhancement in the brain and meninges. Radiographics 2007;27:525-51 CrossRef Medline

15. Wen L, Hu LB, Feng XY, et al. Rathke's cleft cyst: clinicopathological and MRI findings in 22 patients. Clin Radiol 2010;65:47-55 CrossRef Medline

16. Naganawa S, Koshikawa T, Nakamura T, et al. Comparison of flow artifacts between 2D-FLAIR and 3D-FLAIR sequences at $3 \mathrm{~T}$. Eur Radiol 2004;14:1901-08 CrossRef Medline

17. Chagla GH, Busse RF, Sydnor R, et al. Three-dimensional fluid attenuated inversion recovery imaging with isotropic resolution and nonselective adiabatic inversion provides improved three-dimensional visualization and cerebrospinal fluid suppression compared to two-dimensional FLAIR at 3 Tesla. Invest Radiol 2008;43:547-51 CrossRef Medline

18. Kallmes DF, Hui FK, Mugler JP, III. Suppression of cerebrospinal fluid and blood flow artifacts in FLAIR MR imaging with a singleslab three-dimensional pulse sequence: initial experience. Radiology 2001;221:251-55 CrossRef Medline

19. Bink A, Schmitt M, Gaa J, et al. Detection of lesions in multiple sclerosis by 2D FLAIR and single-slab 3D FLAIR sequences at 3.0 T: initial results. Eur Radiol 2006;16:1104-10 CrossRef Medline 